\title{
Structure and Functional Properties of Antioxidant Nanoemulsions Prepared with Tea Polyphenols and Soybean Protein Isolate
}

\author{
Bo Tian', Yixiao Wang ${ }^{1}$, Tuanjie Wang ${ }^{1}$, Lijing Mao' ${ }^{1}$, Yini Lu¹, Huiting Wang ${ }^{1}$, and \\ Zhibiao Feng ${ }^{2 *}$ \\ ${ }_{1}^{1}$ Food Science College, Northeast Agricultural University, Harbin 150030, CHINA \\ ${ }^{2}$ Chemistry College, Northeast Agricultural University, Harbin 150030, CHINA
}

\begin{abstract}
In this study, tea polyphenols (TP) was added to a soy protein isolate (SPI) to prepare nanoemulsions by ultra-high pressure homogenization (UHPH). The nanoemulsions were characterized by a confocal laser scanning electron microscopy, infrared spectroscopy, dynamic rheometer and size-potential analyzer. The effects of TP on the hydrophobicity, emulsifiability, particle size, potential and antioxidant capacity of the prepared nanoemulsions were investigated. The properties of the nanoemulsions with different concentrations of TP were analyzed. The results indicated that ultra-high pressure homogenization treatment contributed to the formation of the SPI-TP complex that showed higher antioxidant activity. The nanoemulsions with good emulsifying properties and high DPPH scavenging ability at the concentration of TP ranged from 0.15-0.20g/ $\mathrm{mL}$. Furthermore, nanoemulsions prepared in this way also had a uniform particle size. Therefore, this nanoemulsions exhibited a good potential to act as an efficient emulsifier.
\end{abstract}

Key words: ultra-high pressure homogenization (UHPH), antioxidant, nanoemulsions, complex

\section{Introduction}

$\mathrm{TP}$ is an important bioactive ingredient in tea, and it is composed of flavanols, lutein, phenolic acids, anthocyanins and glycolic acid ${ }^{1)}$. TP has attracted people's attention because of its wide application in the health care industry ${ }^{2}$. Its biological activities include antioxidant, free radical scavenging, inhibition of lipid peroxidation, anti-inflammatory and other properties. Recently, studies using various in vitro and animal models have shown that TP is one of the most commonly used antioxidants and plays an important role in delaying the onset or stopping the progression of neurodegenerative diseases ${ }^{3)}$. Zhang et al. found that the main active compound in green tea is catechins (flavanols), in which epigallocatechin gallate (EGCG) is the most active compound ${ }^{4)}$. EGCG is widely found in plants as a naturally occurring compound, and animal experiments have shown that EGCG has a good anticancer effect ${ }^{5)}$.

Soy protein isolate (SPI) is a mixture of proteins. extracted from soybean meal as a raw material by dissolving in an alkaline solution, precipitation under acidic conditions, and reprocessing to obtain a protein content of more than $90 \%{ }^{6}$. SPI has various properties that make it suitable as the raw material to make nanoemulsions. The charge nature of the molecule and the presence of hydrophilic and hydrophobic groups in the molecule allows the protein to adsorb at the oil-water interface. The protein can form a barrier film around the oil droplets ${ }^{7}$. As other food protein components, SPI has the ability to reduce the interfacial tension in the emulsion system ${ }^{8}$. Heat treatment favors the formation of the SPI-resveratrol complex and imparts higher antioxidant activity, whereas water solubility of resveratrol by complexation with SPI was also enhanced mainly due to hydrophobic interaction ${ }^{9)}$. However, due to its low surface hydrophobicity, large molecular size and low molecular flexibility ${ }^{10)}$, SPI cannot adsorb rapidly to the oil-water interface. The emulsifying ability of a protein largely depends on the molecular structure and the physicochemical properties of the protein ${ }^{11}$. It is generally considered to be a less efficient emulsifier than other proteins.

A uniform mixture with droplet sizes in the range 10-500 $\mathrm{nm}$ formed from two or more immiscible liquids is known as nanoemulsion ${ }^{12)}$. Compared with conventional emulsions, nanoemulsions used as delivery systems have many advantages, such as a high optical clarity, a good physical

\footnotetext{
*Correspondence to: Zhibiao Feng, Chemistry College, Northeast Agricultural University, Harbin 150030, CHINA

E-mail: fengzhibiao@neau.edu.cn

Accepted April 16, 2019 (received for review March 10, 2019)

Journal of Oleo Science ISSN 1345-8957 print / ISSN 1347-3352 online

http://www.jstage.jst.go.jp/browse/jos/ http://mc.manusriptcentral.com/jjocs
} 
and chemical stability, and bioavailability ${ }^{13)}$. When nanoemulsions are used as a delivery system for bioactive lipids, drugs, fragrances, antioxidants and other functional components, it greatly improves the water solubility, stability and bioavailability of the entire system ${ }^{14)}$. Studies have shown that many food proteins have a stabilising emulsion capacity similar to conventional surfactants, such as bovine serum albumin (BSA), whey protein isolate(WPI), and $\mathrm{SPI}^{15)}$.

In addition, it has been reported that proteins can bind to polyphenols through covalent bonds, hydrophobic interactions, etc. ${ }^{16-18)}$. They interact mainly through noncovalent bonds and hydrophobic interactions followed by stable hydrogen bonds between proteins and polyphenols ${ }^{19)}$. TP can be combined with $\beta$-and $\alpha$-casein by hydrophilic and hydrophobic properties, but hydrophobic binding plays the main role ${ }^{20)}$. Another study has reported on the use of these extracts added in model olive oil-in-water $(\mathrm{O} / \mathrm{W})$ emulsions to study their effects on physical and chemical stability. The rheological behaviour and creaming stability of the emulsions were dramatically improved by using xanthan gum $^{21)}$. A further studied also modelled the behaviour of these types of $\mathrm{O} / \mathrm{W}$ emulsions trying to generalise their physical and chemical stability. Limited hydrolysis of $7 \mathrm{~S}$ (a component of soy protein isolate) via trypsinization, at as low as degrees of hydrolysis of $1 \%$, helped to improve emulsion oxidative stability at $\mathrm{pH} 7$ under low ionic strength $^{22)}$. Staszewski et al. studied the complexation of tea polyphenols and $\beta$-lactoglobulin. The results showed that several amino acid residues of proteins involved in the formation of polyphenol-protein complexes were bound by hydrophilic and hydrophobic interaction ${ }^{23)}$. Moreover, the appearance of the structure of the larger particles is formed by a hydrophobic interaction between $\beta$-lactoglobulin or casein polypeptide and tea polyphenol ${ }^{24)}$. Studies have shown that partially opening the structure of SPI improves its emulsification ${ }^{25}$. Ultrahigh pressure homogenization (UHPH) is a new technology for changing the structure of proteins, and it is also a way to prepare stable submicron emulsions ${ }^{26,27)}$. UHPH produces intense turbulence, vibration, cavitation and hydraulic shear. These forces can break down droplets into nanodroplets ${ }^{28)}$. UHPH emulsions treated with 100 and $200 \mathrm{MPa}$ are the most stable because of their lower particle size, greater viscosity, and partial denaturation ${ }^{29)}$.

In the present study, TP and SPI were combined by high pressure homogenization to prepare nanoemulsions. The nanoemulsions was adsorbed on the surface of oil as an emulsifier to enhance the antioxidant effect of droplets. The effects of different TP concentrations on microstructure and function of the nanoemulsions were studied. Nanoemulsions can be used as carriers to transport medicines and cosmetics. The study has certain significance for the application of oxidizable substances in food and pharma- ceutical industries.

\section{Chemicals and Reagents 2.1 Materials}

SPI was purchased from Zhejiang Qianyu Biotechnology Co., Ltd, China. $\mathrm{KBr}$ (with purity of $99.0 \%$ ) was supplied by Shanghai Macklin Biochemical Co., Ltd. Tea Polyphenols (purity, 97.0\%) was provided by Zhongcheng Chemical Co., Ltd, China. Tea Polyphenols exhibited yellow powder with good solubility in water. The peanut oil ( $41 \%$ oleic acid, $37 \%$ linoleic acid, $20 \%$ of saturated fatty acids such as palmitic acid, stearic acid and arachidic acid.) was purchased from the local market (Harbin, China). 1-phenylamino-8-naphthalenesulfonic acid(ANS), 1,1-diphenyl2-trinitrophenylhydrazine (DPPH) and Sodium dodecyl sulfonate(SDS) were obtained from Sigma Chemical Co. (St. Louis, MO, USA). All the chemicals used were of analytical grade and were used without any further purification.

\subsection{Preparation of nanoemulsions}

In our test, the viscosity of SPI solution was too large when SPI concentration was higher than $1.0 \mathrm{~g} / 100 \mathrm{~mL}$. However, when the SPI concentration was less than 1.0 $\mathrm{g} / 100 \mathrm{~mL}$, the prepared emulsions had less emulsifying. Therefore, the concentration of SPI was used at $1.0 \mathrm{~g} / 100$ $\mathrm{mL}$. The SPI solution was prepared at a concentration of $1.0 \mathrm{~g} / 100 \mathrm{~mL}$ with phosphate buffer $(0.01 \mathrm{M}, \mathrm{pH} 7.0)$ and was magnetic stirred at room temperature for $3 \mathrm{~h}$. The $\mathrm{pH}$ was adjusted to 7.0 with either $\mathrm{HCl}$ or $\mathrm{NaOH}$. The solution was then placed in a $4^{\circ} \mathrm{C}$ refrigerator overnight to ensure complete dispersion. TP was added at concentrations of $0.00,0.10$, or $0.20 \mathrm{~g} / 100 \mathrm{~mL}$ (TP/Protein solution) at room temperature. Peanut oil was added according to the ratio of SPI to oil at $3: 1(\mathrm{v} / \mathrm{v})$. The primary emulsions were archived with a high-speed dispersion machine (Omni GLH-220 High Speed Homogenizer, U.S.A.) at 10000 rpm for $5 \mathrm{~min}$, which were further homogenized twice with an ultrahigh pressure homogenizer (100 Mpa) (Stansted Fluid Power, SPCH-10, United Kingdom) to make the microemulsion nanometer liquid ${ }^{30)}$.

\section{Determination of functional properties of nanoemulsions \\ 3.1 Determination of surface hydrophobicity}

The hydrophobicity of the emulsions was detected by the ANS fluorescence probe method ${ }^{31)}$. The nanoemulsion sample was diluted to $0.2 \mathrm{~g} / 100 \mathrm{~mL}, 0.1 \mathrm{~g} / 100 \mathrm{~mL}, 0.05$ $\mathrm{g} / 100 \mathrm{~mL} 0.01 \mathrm{~g} / 100 \mathrm{~mL}$ with phosphate buffer $(0.01 \mathrm{M}, \mathrm{pH}$ 7.0 ), and $25 \mu \mathrm{L}$ of an $8 \mathrm{mmol} / \mathrm{L}$ ANS solution was added to $6 \mathrm{~mL}$ of the sample solution with different concentrations. 
The solutions were kept for 15 min after mixing thoroughly. The fluorescence intensity was measured using a F-4500 Fluorescence Spectrometer (Shimadzu Corporation, Japan). The excitation wavelength $\lambda \mathrm{ex}=390 \mathrm{~nm}$, the emission wavelength $\lambda \mathrm{em}=470 \mathrm{~nm}$, and the gap was $5 \mathrm{~nm}$. With the fluorescence intensity as the ordinate, the sample concentration for the abscissa plot and the slope value of the initial segment were the surface hydrophobicity of the protein molecule.

\subsection{Emulsion activity and emulsion stability of nanoemul- sions}

The determination of the emulsion activity of the nanoemulsions was based on a previous study. Briefly, $10 \mu \mathrm{L}$ of nanoemulsions at the bottom of the solution was taken at 0 min and $60 \mathrm{~min}$. Each was supplemented with $6 \mathrm{~mL}$ of a $0.1 \%$ SDS solution and was mixed thoroughly. The absorbance was measured at $500 \mathrm{~nm}$, with $0.1 \%$ SDS as the blank. The emulsion activity index (EAI) and emulsion stability index (ESI) were determined based on the formulas in equations (1) and (2) as previously reported ${ }^{32)}$.

$$
\begin{aligned}
& \operatorname{EAI}\left(\mathrm{m}^{2} / \mathrm{g}\right)=2 \mathrm{~T} \frac{A_{0} \times N}{C \times \Phi \times 1000} \\
& \operatorname{ESI}(\mathrm{min})=\frac{A_{0}}{A_{0}-A_{1}} \times t
\end{aligned}
$$

where $\mathrm{T}=2.303, A_{0}$ is the absorbance of the nanoemulsions immediately, $\mathrm{N}$ is the dilution factor $(600 \times)$, c is the weight of the SPI per volume $(\mathrm{g} / \mathrm{mL}), \Phi$ is the oil volume fraction of the emulsion, $A_{1}$ is the absorbance of the nanoemulsions at $60 \mathrm{~min}$,and $t$ is the time interval, $60 \mathrm{~min}$.

\subsection{Antioxidant activity of the nanoemulsions using the DPPH assay}

The preparation of the DPPH $(0.1 \mathrm{mM})$ ethanol solution was carried out according to Li et al. in $2012^{33)}$. Briefly, $19.7 \mathrm{mg}$ DPPH powder was accurately weighed and dissolved in $500 \mathrm{~mL}$ absolute ethanol and was stored at $4^{\circ} \mathrm{C}$. Before the measurement, $2.9 \mathrm{~mL}$ of the DPPH solution prepared previously was added to $0.1 \mathrm{~mL}$ of the nanoemulsions. The mixture was incubated in the dark at room temperature for $30 \mathrm{~min}$, with absolute ethanol as the blank at $517 \mathrm{~nm}$ measured absorbance $A_{0}, 0.1 \mathrm{~mL}$ of deionized water instead of the nanoemulsions absorbance $A_{1}$, and 2.9 $\mathrm{mL}$ of absolute ethanol instead of the DPPH anhydrous ethanol solution absorbance $A_{2}$, according to the following formula to calculate the clearance rate:

$$
\mathrm{DPPH} \text { clearance rate }(\%)=\left(1-\frac{A_{0}-A_{2}}{A_{1}}\right) \times 100
$$

\subsection{Determination of viscosity}

The viscosity of the nanoemulsions was measured according to the method described in previous study with a slight improvement ${ }^{34)}$. The rheological measurements were carried out using a dynamic rheometer (Switzerland Buchi company). The emulsion viscosity was measured at $25^{\circ} \mathrm{C}$, over a shear rate range of $0.01-100 \mathrm{~s}^{-1}$, with cone-plate geometry $\left(\mathrm{CP} 40 / 4^{\circ}\right)$.

3.5 Particle size, distribution, polydispersity index(PDI), and Zeta-potential (ZP) measurement

A laser particle size analyzer (Msatersizer 2000) was used to detect the particle size of nanoemulsions, and the analyzer can simultaneously detect the PDI value and particle size of the nanoemulsions. The emulsion sample was normally diluted before measurement because of low scattering intensity. For this experiment, the appropriate amount of the composite emulsion was added to the dispersant (water) to obtain a protein concentration of $1 \mathrm{~g} / 100 \mathrm{~mL}$, and approximately $1 \mathrm{~mL}$ of the sample was placed into the particle size measuring cup, and the incident light angle of the analyzer and other parameters to start measuring the particle size and PDI value were adjusted. The samples were placed in a potential measuring cup to determine their potential. All the measurements were carried out at $25^{\circ} \mathrm{C}$ in triplicate.

\subsection{Nanoemulsions microstructure characterization}

The microstructure of the emulsions was measured using a confocal laser scanning microscope (CLSM, Leica Microsystems Inc., Heidelberg, Germany) with a $100 \times$ oil immersion objective lens. For the staining, $40 \mu \mathrm{L}$ of the staining solution ( $0.1 \%$ Nile Red and $0.1 \%$ Nile Blue) and $1 \mathrm{~mL}$ of nanoemulsions were mixed, then keep them for 20 minutes in dark. The Nile Red stained the oil, and the Nile Blue A stained the protein. The nanoemulsions $(10 \mu \mathrm{L})$ were placed on concave confocal microscope slides. It was examined using an argon Krypton Laser (ArKr, 488 nm) and a Helium Neon laser (HeNe, 633 nm). The oil phases appeared red, and the protein was green ${ }^{35)}$.

\subsection{Infrared spectroscopy}

Nanoemulsions samples were prepared with a TP concentration of $0.20 \mathrm{~g} / 100 \mathrm{~mL}$. A sample without TP was used as a control, and then, all the samples were dried under vacuum. $2 \mathrm{mg}$ samples were mixed with $200 \mathrm{mg} \mathrm{KBr}$ to compress the powder ${ }^{36)}$. Interferograms were accumulated over the spectral range of $4000-400 \mathrm{~cm}^{-1}$ with a nominal resolution of $2 \mathrm{~cm}^{-1}$ and 64 scans.

\subsection{Statistical analysis}

The data were processed and analyzed by Origin 8.0 and SPSS 19.0 software. The data are expressed by $X \pm S E$, and $p<0.05$ was considered significant. Unless specified otherwise, three independent trials were performed, and each with a new batch of sample prepared. 


\section{Results and Analysis}

\subsection{TP effects of concentrations on SPI hydrophobicity}

Figure 1 showed the hydrophobicity of SPI in TP solutions at different concentrations. With the increased of TP concentration, the surface hydrophobicity of SPI gradually decreased. The protein hydrophobicity was determined by the number of hydrophobic groups on the surface that came into contact with a polar aqueous environment ${ }^{36)}$. There were many hydrophilic hydroxyl groups on the surface of TP, and it combined with SPI to form a complex, thereby increasing the surface hydrophilicity of the SPI. When the concentration of TP was between 0.05-0.20 g/100 $\mathrm{mL}$, the surface hydrophobicity of SPI was not significantly different $(p>0.05)$. The combination of TP and SPI occurred through hydrophobic interactions, which reduced the hydrophobic groups on the SPI surface, and thus, the surface hydrophobicity of the SPI was decreased. This was consistent with the results of previous studies, showing that the mutual binding of polyphenols and proteins is achieved through hydrophobic interactions ${ }^{37)}$. The result also supported the conclusion that the binding of tea polyphenols to $\beta$-casein and $\alpha$-casein was also achieved through hydrophobic interaction ${ }^{38)}$.

\subsection{Emulsion activity index (ESI) and emulsion stability}

The emulsifying characteristics were related to the denaturation of proteins and their decreased solubility ${ }^{39)}$. The effect of different concentrations of TP on the ESI and emulsion stability of nanoemulsions were shown in Fig. 2. From the Fig. 2, as the TP concentration gradually increased, ESI of the nanoemulsions did not change and lacked regularity. The emulsion stability of the nanoemulsions also increased first and then decreased with the increased TP concentration. When the concentration of TP was in the range of $0.00-0.20 \mathrm{~g} / 100 \mathrm{~mL}$, the emulsion stability increased as the concentration went up. When the TP

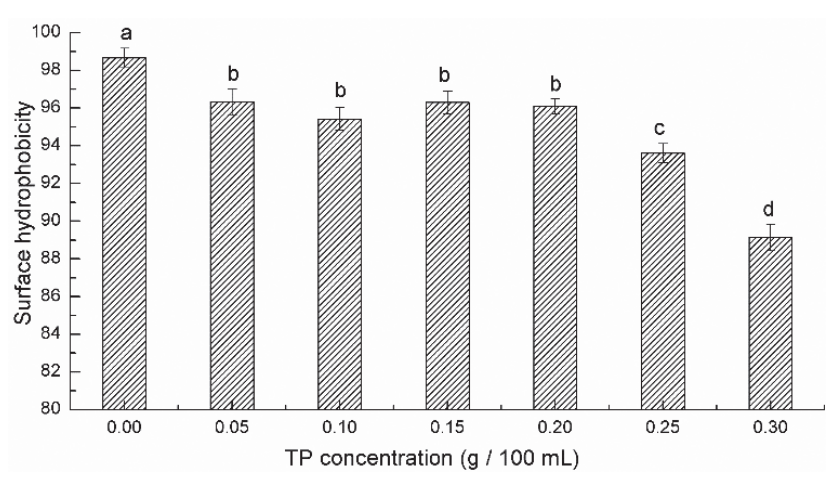

Fig. 1 The effect of different concentrations of TP on hydrophobicity of SPI.

The same letters indicated no significant differences and different letters represented significant differences, and the same below. concentration was between 0.20 and $0.25 \mathrm{~g} / 100 \mathrm{~mL}$, the emulsification stability was the best, but there was no significant difference $(p>0.05)$. When the TP concentration increased from 0.25 to $0.30 \mathrm{~g} / 100 \mathrm{~mL}$, the emulsion stability significantly declined. We assumed that in the presence of TP, the structure of SPI changed partially/totally after UHPH. As the combination of TP and SPI increased the phenolic hydroxyl group on the surface of the SPI, the hydrophilicity of the protein that adsorbed on the surface of the oil droplet in the nanoemulsions was enhanced. When the nanoemulsions formed, the oil droplets were stable and present in the water phase and did not easily aggregate into large particles, further enhancing the emulsion stability of the nanoemulsions. When the TP concentration was less than $0.25 \mathrm{~g} / 100 \mathrm{~mL}$, the emulsion stability of the nanoemulsions gradually increased with the increased TP concentration. However, when the TP concentration was more than $0.25 \mathrm{~g} / 100 \mathrm{~mL}$, the polyphenols and protein combined to form a small amount of insoluble complex. The result was consistent with previous research reports ${ }^{40)}$. The emulsification of SPI was reduced, which reduced the emulsion stability of the nanoemulsions. In comparison, the emulsion stability of nanoemulsions was more important than the ESI. Therefore, the concentration of the TP was the main index, and the concentration of the TP should be between $0.10-0.25 \mathrm{~g} / 100 \mathrm{~mL}$.

\subsection{Effects of different concentrations of TP on DPPH scavenging ability of nanoemulsions}

DPPH was a stable group and had been widely used to test the free radical scavenging effects of various antioxidants $^{41,42)}$. The effect of the TP concentration on the ability of the compound nanoemulsions system to remove DPPH was shown in Fig. 3. With the increased TP concentration, the ability to reduce DPPH increased and then decreased.

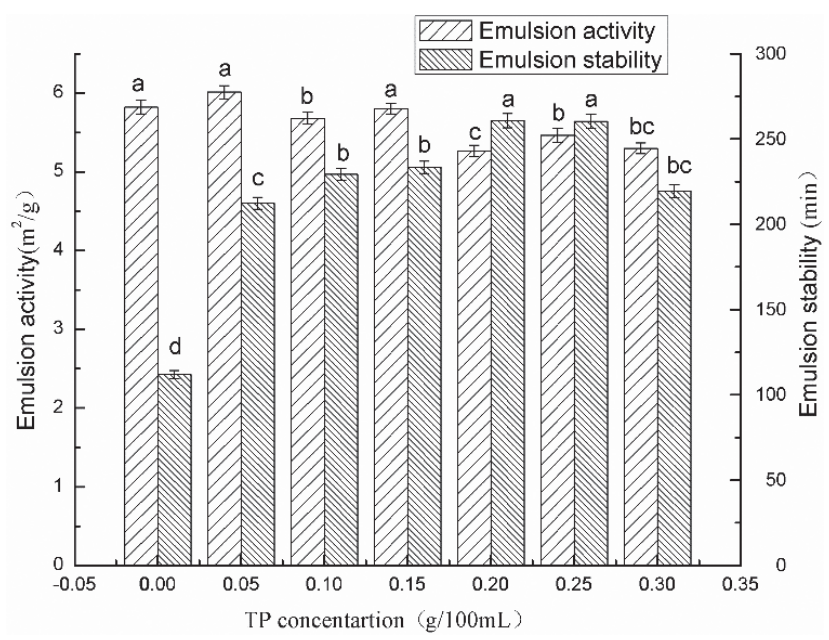

Fig. 2 The ESI and EAI emulsifications with different concentration of TP. 


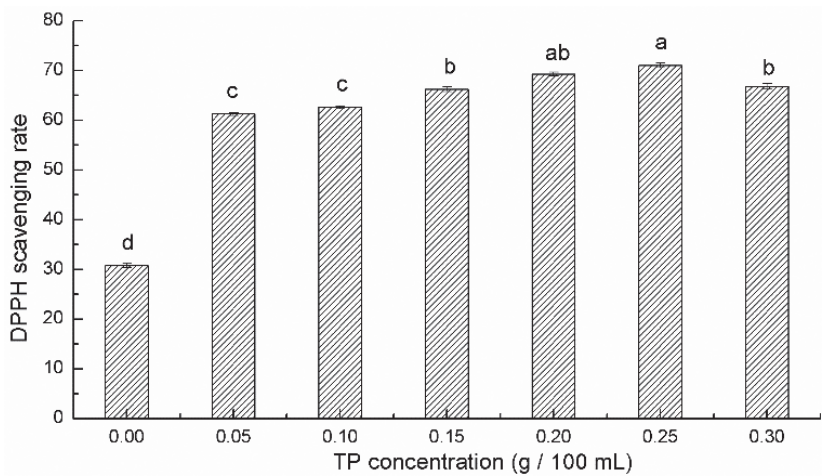

Fig. 3 The effect of different concentration of TP on DPPH scavenging ability of nanoemulsions.

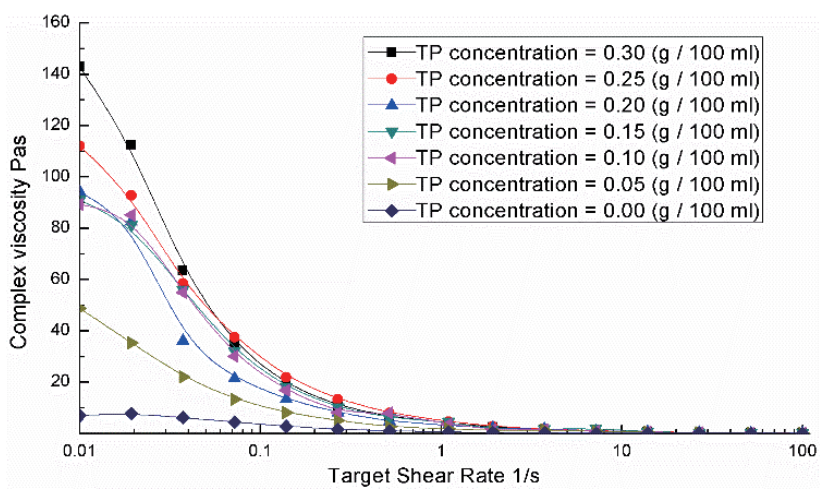

Fig. 4 The effect of different concentration of TP on the viscosity of nanoemulsions.

Table 1 The effect of different concentration of TP on the potential, particle size and PDI vale of nanoemulsions.

\begin{tabular}{cccc}
\hline TP concentration $(\mathrm{g} / 100 \mathrm{~mL})$ & Potential $(\mathrm{mV})$ & Particle size $(\mathrm{nm})$ & PDI value \\
\hline 0.00 & -37.5 & 800 & 0.54 \\
0.05 & -39.5 & 350 & 0.30 \\
0.10 & -39.8 & 360 & 0.28 \\
0.15 & -43.2 & 380 & 0.36 \\
0.20 & -42.5 & 340 & 0.35 \\
0.25 & -41.0 & 345 & 0.43 \\
0.30 & -39.5 & 390 & 0.29 \\
\hline
\end{tabular}

The particle sizes and PDI values are based on intensity distribution.

The results of a study on the antioxidant capacity of tea polyphenols combined with proteins is presented by Hajieva et $a l .{ }^{16)}$. When the TP concentration was between 0.05 and $0.10 \mathrm{~g} / 100 \mathrm{~mL}$, the DPPH scavenging ability was not significantly changed $(p>0.05)$. However, it was worth noting that when the tea polyphenol concentration was increased from 0.10 to $0.15 \mathrm{~g} / 100 \mathrm{~mL}$, the antioxidant activity of the nanoemulsions was significantly improved, and the nanoemulsions with TP concentration of $0.20 \mathrm{~g} / 100 \mathrm{~mL}$ and $0.25 \mathrm{~g} / 100 \mathrm{~mL}$ had little difference in antioxidant activity. When the TP concentration reached $0.30 \mathrm{~g} / 100 \mathrm{~mL}$, the DPPH scavenging ability was significantly decreased $(p<$ $0.05)$. Considering the emulsifying activity, emulsifying stability and reducing power of the nanoemulsions, the TP concentration should be between 0.15 and $0.25 \mathrm{~g} / 100 \mathrm{~mL}$. The information obtained in these results will facilitate the uses and applications of nutraceuticals-loaded nanoemulsions delivery system ${ }^{43)}$.

\subsection{The relationship between the concentration of TP and the viscosity of nanoemulsions}

The viscosities of the nanoemulsions with $\mathrm{TP}$ at different concentrations were shown in Fig. 4. With an increased shear rate, the viscosity of the nanoemulsions gradually decreased, indicating that the nanoemulsions conformed to exhilited a shear thinning behavior. When the shear rate was $0.01 \mathrm{~s}^{-1}$, the viscosity of the nanoemulsions increased with the increased TP concentration. At the same shear rate, the addition of TP increased the viscosity of the nanoemulsions compared to the soy protein nanoemulsions. The reason was that TP and SPI were combined to form a TP-SPI complex by hydrophobic interaction, and TP contained a large amount of hydroxyl groups, eventually leading to an increase in viscosity of the nanoemulsions ${ }^{44)}$. In addition, the emulsion stability of nanoemulsions was related to its viscosity. The emulsion stability of the nanoemulsions gradually increased with the increased viscosity.

\subsection{Effect of TP concentration on particle size, distribu- tion, PDI, and zeta potential}

The effect of the TP concentration on the size and the PDI of the nanoemulsions particles were shown in Table 1. The particle size of the nanoemulsions added with TP was significantly lower than the nanoemulsions without TP. However, when the TP concentration was $0.30 \mathrm{~g} / \mathrm{mL}$, the particle size of the nanoemulsions was significantly increased $(p<0.05)$. With a TP concentration between 0.05 $0.25 \mathrm{~g} / 100 \mathrm{~mL}$, the nanoemulsions particle size was smaller. PDI is an indicator to evaluating the dispersion of nanoemulsions. When TP was added, the PDI of the nano- 


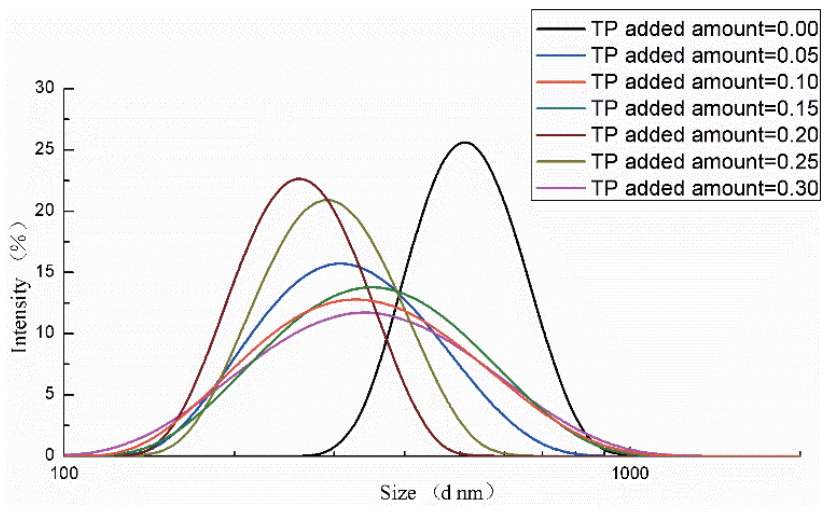

Fig. 5 The distribution of nanoemulsions particle size adding different amount of TP.

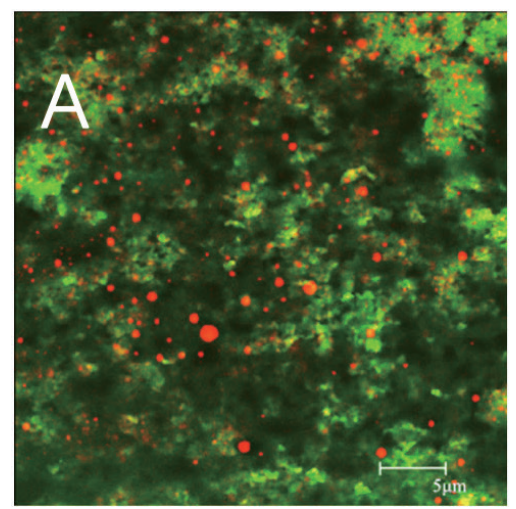

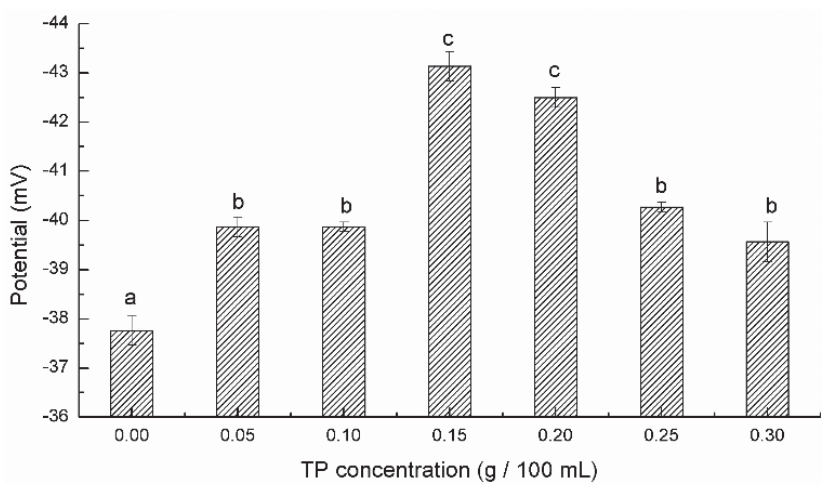

Fig. 6 The zeta potential values of nanoemulsions adding different amount of TP.

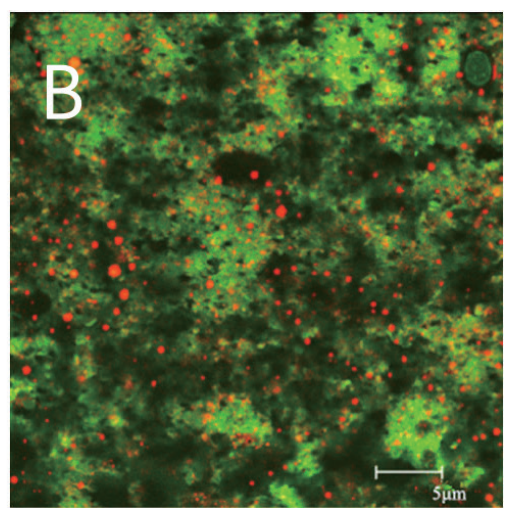

Fig. 7 The laser confocal microscope images of different nanoemulsions.

$\mathrm{A}$ and $\mathrm{B}$ indicate that the nanoemulsions with TP concentrations are $0 \mathrm{~g} / 100 \mathrm{~mL}$, and $0.15 \mathrm{~g} / 100 \mathrm{~mL}$.

emulsions was significantly lower than that of the nanoemulsions without TP. The lower PDI indicated better dispersion. With the change in TP concentration, the PDI of the nanoemulsions was not significantly different $(p<$ 0.05). Adding TP reduced the PDI and the particle size distribution of the nanoemulsions. When the amount of TP added was between $0.05-0.30 \mathrm{~g} / 100 \mathrm{~mL}$, the particle size distribution of the nanoemulsions was relatively uniform.

From the intensity distribution of the particle sizes in Fig. 5, it was demonstrated that when the TP concentration was low, the particle size distribution was larger, and as the concentration of TP increases, the particle size distribution gradually moved to a small range and tended to be stable.

The emulsion stability of the nanoemulsions was positively correlated with the absolute value of the potential. Figure 6 showed that when the concentration of tea polyphenol increased from 0 to $0.15 \mathrm{~g} / \mathrm{mL}$, the absolute value of emulsion potential gradually increased. While the TP concentration exceeded $0.15 \mathrm{~g} / \mathrm{mL}$, the absolute value of emulsion potential slowly decreased. When the TP concentration reached $0.15 \mathrm{~g} / 100 \mathrm{~mL}$, the potential reached the maximum value of $-43.2 \mathrm{mV}$, and the nanoemulsions had the best stability.
TP has many hydrophilic hydroxyl groups. When it formed a complex with SPI, the surface hydrophilicity of the SPI was enhanced, and the complex wrapped on the surface of the oil droplets and enhanced the interaction between the emulsion surface and water, enhanced the viscosity of the nanoemulsions eventually. Thereby, it reduced the particle size of the nanoemulsions and lowed their PDI, making the nanoemulsions uniform. After the addition of $\mathrm{TP}$, the surface charge of the SPI increased significantly, thus, it was easier to protonate to the negatively charged surface of $\mathrm{TP}^{45)}$. Therefore, the absolute value of the surface charge of the nanoemulsions increased, and the nanoemulsions were more stable.

\subsection{Observation on microstructure of nanoemulsions with different concentration of TP}

Figure 7 showed the observation of the different concentrations of tea polyphenol emulsions using a confocal laser scanning microscope. When the TP was not added, the nanoemulsions exhibited large particle size, and the particle size gradually decreased with the increased of TP concentration. The nanoemulsions with the TP added had a more uniform particle size distribution and smaller particle size than the nanoemulsions without TP. It can also be seen 

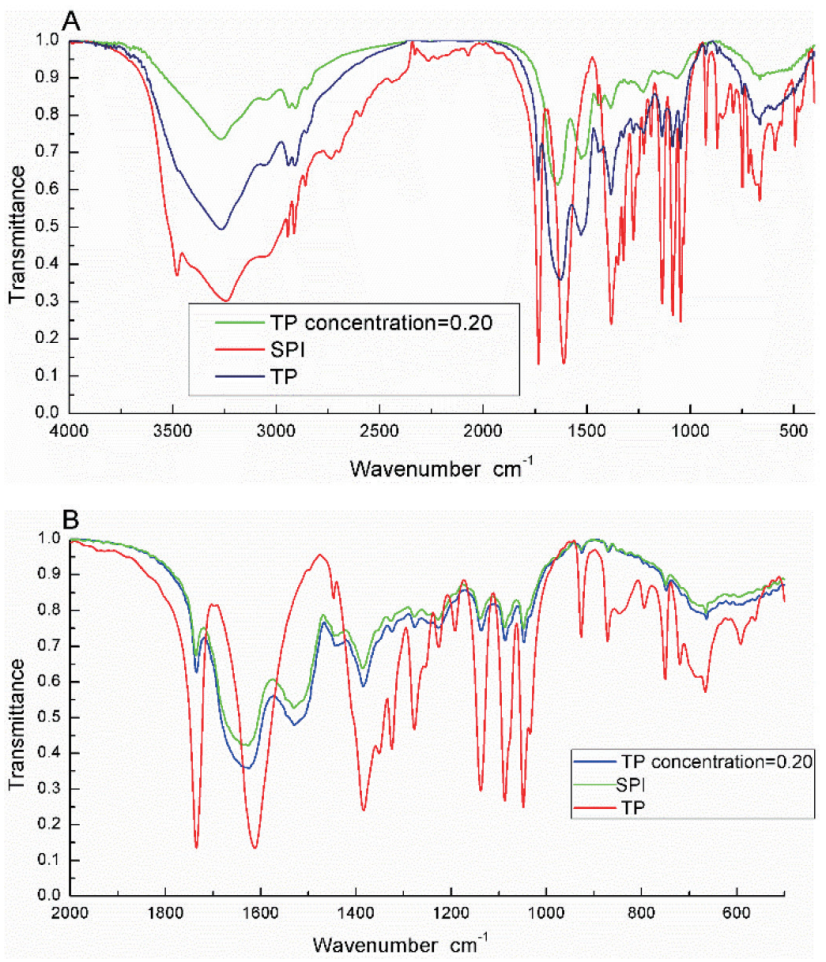

Fig. 8 Different concentrations of TP infrared spectra. A is the full wavelength of $4000 \mathrm{~cm}^{-1}-400 \mathrm{~cm}^{-1}$ infrared spectrum B is the full wavelength of 2000 $\mathrm{cm}^{-1}-500 \mathrm{~cm}^{-1}$ infrared spectrum.

from the figure that the particle size of the nanoemulsions was less than $1 \mu \mathrm{m}$, which was consistent with the results of the particle size analyzer. Thus, TP improved the particle size distribution of the nanoemulsions.

\subsection{Infrared spectroscopy}

The coupling between the hydrogen bond and the dipole was a key factor in controlling the protein conformationally sensitive regional amide band, amide I with $1700-1600 \mathrm{~cm}^{-1}$ (mainly $\mathrm{C}=\mathrm{O}$ stretching vibration) and amide II with 1600$1500 \mathrm{~cm}^{-1}$ (Mainly C-N stretching coupling and $\mathrm{N}-\mathrm{H}$ bending vibration). Protein amides I and II were associated with structural changes in proteins ${ }^{46}$. Figure 8 showed that the amide I band of the SPI shifted from $1640 \mathrm{~cm}^{-1}$ to $1624 \mathrm{~cm}^{-1}$ after adding TP, and the amide II band shifted to $1526 \mathrm{~cm}^{-1}$ from $1530 \mathrm{~cm}^{-1}$, indicating that the secondary structure of SPI had changed ${ }^{38)}$. Additionally, an increased intensity was observed for the protein amide I and amide II in the different spectra of the TP-SPI complexes. The increase in the intensity of the amide I and amide II bands was due to the polyphenol binding to the protein $\mathrm{C}$ $=\mathrm{O}, \mathrm{C}-\mathrm{N}$ and $\mathrm{N}-\mathrm{H}$ groups (hydrophilic interaction) ${ }^{47}$. Similar infrared spectral changes were observed for protein amide I band in several ligand-protein complexes, where major protein conformational changes occur ${ }^{48)}$.

The broad peak at $3244 \mathrm{~cm}^{-1}$ in the infrared spectrum represents the alcohol hydroxyl peak of the hydroxyl interaction of TP. Figure $8 \mathrm{~A}$ showed that after adding TP, the maximum peak of the SPI here was from $3267 \mathrm{~cm}^{-1}$ to $4358 \mathrm{~cm}^{-1}$, which was due to the interaction between the SPI and TP phenolic hydroxyl functional groups. This change was consistent with Xiaojing Li et al. ${ }^{49}$ which demonstrated that polysaccharide starch forms a complex with peanut protein. $\mathrm{C}=\mathrm{O}$ stretching vibration produces a 1735 $\mathrm{cm}^{-1}$ absorption peak, and C-O stretching vibration produces a $1385 \mathrm{~cm}^{-1}$ and $1324 \mathrm{~cm}^{-1}$ absorption peak, C-O elongation yielded $1226 \mathrm{~cm}^{-1}, 1136 \mathrm{~cm}^{-1}, 1087 \mathrm{~cm}^{-1}, 1047$ $\mathrm{cm}^{-1}$ absorption peak, and a peak similar to TP was observed on the TP-SPI complex but was not seen on the SPI spectrum.

\section{Conclusions}

In this study, the physicochemical properties of the SPI nanoemulsions with different concentrations of TP were investigated. The results showed that after high pressure homogenization, TP formed a complex with SPI, which changed the secondary structure of the SPI. The spatial structure of the SPI was more stretched and the hydrophobicity decreased, which further improved the emulsion stability of the nanoemulsions. Meanwhile, compared with the SPI nanoemulsions, the addition of TP made the nanoemulsions smaller in particle size and more uniform in distribution, which further increased the stability of the nanoemulsions. The prepared TP-SPI nanoemulsions possessed good dispersion and stability. The proposed method could be a novel way of utilizing plant proteins in the long-term stabilization of nanoemulsions in the food and beverage industry $^{50)}$. The nanoemulsions prepared in this paper can be used as a protective agent to embed certain biologically active substances in them to protect the biological activity.

\section{Acknowledgments}

We thank all authors for assisting in preparation of this manuscript and the authors have declared that no conflict of interest exists.

\section{Reference}

1) Pietta, P.G. Flavonoids as antioxidants. J. Nat. Prod. 63, 1035-1042 (2000).

2) Narendra, K.; Ajay, M. Thermodynamic and physicochemical properties evaluation for formation and characterization of oil-in-water nanoemulsion. J. Mol. Liq. 266, 147-159 (2018).

3) Caruana, M.; Vassallo, N. Tea polyphenols in Parkin- 
son's disease. Adv. Exp. Med. Biol. 863, 117-137 (2015).

4) Zhang, L.; Wei, Y.; Zhang, J. Novel mechanisms of anticancer activities of green tea component epigallocatechin- 3-gallate. Anticancer Agents Med. Chem. 14, 779-786 (2014).

5) Shin, Y.S.; Kang, S.U.; Park, J.K.; Kim, Y.E.; Kim, Y.S.; Baek, S.J.; Lee, S.H.; Kim, C.H. Anti-cancer effect of ( - )-epigallocatechin-3-gallate (EGCG) in head and neck cancer through repression of transactivation and enhanced degradation of $\beta$-catenin. Phytomedicine 23, 1344-1355 (2016).

6) Kumar, R.; Choudhary, V.; Mishra, S.; Varma, I.K.; Bo, M. Adhesives and plastics based on soy protein products. Ind. Crops Prod. 16, 155-172 (2002).

7) Phoon, P.Y.; Narsimhan, G. Effect of hydrolysis of soy $\beta$-conglycinin on the oxidative stability of O/Wemulsions. Food Hydrocoll. 35, 429-443 (2014).

8) Keerati, M. Heat-induced changes in oil-in-water emulsions stabilized with soy protein isolate. Food Hydrocoll. 23, 2141-2148(2009).

9) Wan, Z.L.; Wang, J.M.; Wang, L.Y.; Yuan, Y.; Yuan, X.Q. Complexation of resveratrol with soy protein and its improvement on oxidative stability of corn oil/water emulsions. Food Chem. 161, 324-331 (2014).

10) Liu, K. Expanding soybean food utilization. Food Technol. 54, 46-59 (2000).

11) Keerati-U-Rai, M.; Miriani, M.; Iametti, S.; Bonomi, F.; Corredig, M. Structural changes of soy proteins at the oil-water interface studied by fluorescence spectroscopy. Colloids Surf. B 93, 41-48(2012).

12) Oca-Ávalos, J.M.M.D.; Candal, R.J.; Herrera, M.L. Colloidal properties of sodium caseinate-stabilized nanoemulsions prepared by a combination of a high-energy homogenization and evaporative ripening methods. Food Res. Int. 100, 143-150 (2017).

13) Xu, J.; Mukherjee, D.; Chang, S. Physicochemical properties and storage stability of soybean protein nanoemulsions prepared by ultra-high pressure homogenization. Food Chem. 240, 1005-1013 (2017).

14) Huang, Q.; Yu, H.; Ru, Q. Bioavailability and delivery of nutraceuticals using nanotechnology. J. Food Sci. 75, 50-57 (2010).

15) Abbas, S.; Hayat, K.; Karangwa, E.; Bashari, M.; Zhang, $\mathrm{X}$. An overview of ultrasound-assisted food-grade nanoemulsions. Food Eng. Rev. 5, 139-157 (2013).

16) Hajieva, P. The Effect of polyphenols on protein degradation pathways: Implications for neuroprotection. Molecules 22, 159 (2017).

17) Staszewski, M.V.; Jara, F.L.; Ruiz, A.L.T.G.; Rosa, J.J.; Carvalho, J.E.; Pilosofa, A.M.R. Nanocomplex formation between $\beta$-lactoglobulin or caseinomacropeptide and green tea polyphenols: Impact on protein gelation and polyphenols antiproliferative activity. J. Funct.
Food 4, 800-809 (2012).

18) Yuksel, Z.; Avci, E.; Erdem, Y.K. Characterization of binding interactions between green tea flavanoids and milk proteins. Food Chem. 121, 450-456 (2010).

19) Jiang, J; Jie, C.; Xiong, Y.L. Structural and emulsifying properties of soy protein isolate subjected to acid and alkaline pH-shifting processes. J. Agric. Food Chem. 57, 7576-83 (2009).

20) Huang, Q.; Yu, H.; Ru, Q. Bioavailability and delivery of nutraceuticals using nanotechnology. J. Food Sci. 75, 50-57 (2010).

21) Caporaso, N.; Genovese, A.; Burke, R.; Barry, R.C.; Sacchi, R. Physical and oxidative stability of functional olive oil-in-water emulsions formulated using olive mill wastewater biophenols and whey proteins. Food Funct. 7, 227-238(2016).

22) Phoon, P.Y.; Narsimhan, G. Effect of hydrolysis of soy $\beta$-conglycinin on the oxidative stability of $\mathrm{O} / \mathrm{W}$ emulsions. Food Hydrocoll. 35, 429-443 (2014).

23) Staszewski, M.V.; Jara, F.L.; Ruiz, A.L.T.G.; Jagus, R.J.; Carvalho, J.E.; Pilosof, A.M.R. Nanocomplex formation between $\beta$-lactoglobulin or caseinomacropeptide and green tea polyphenols: Impact on protein gelation and polyphenols antiproliferative activity. J. Funct. Foods 4, 800-809 (2012).

24) Yuksel, Z.; Avci, E.; Erdem, Y.K. Characterization of binding interactions between green tea flavanoids and milk proteins. Food Chem. 121, 450-456 (2010).

25) Jiang, J.; Jie, C.; Xiong, Y.L. Structural and emulsifying properties of soy protein isolate subjected to acid and alkaline $\mathrm{pH}$-shifting processes. J. Agric. Food Chem. 57, 7576-7583 (2009).

26) Dumay, E.; Chevalier, L.D.; Picart, P.L.; Benzaria, A.; Julià, A.G. Technological aspects and potential applications of (ultra) high-pressure homogenisation. Trends Food Sci. Tech. 31, 13-26 (2013).

27) Hebishy, E.; Buffa, M.; Guamis, B.; Blasco, M.A.; Trujillo, A.J. Physical and oxidative stability of whey protein oil-in-water emulsions produced by conventional and ultra-high-pressure homogenization: Effects of pressure and protein concentration on emulsion characteristics. Innov. Food Sci. Energ. Technol. 32, 79-90 (2015).

28) Zhang, X.; Haque, Z.Z. Generation and stabilization of whey-based monodisperse nanoemulsions using ultrahigh-pressure homogenization and small amphipathic co-emulsifier combinations. J. Agric. Food Chem. 63, 10070-10077 (2015).

29) Fernándezávila, C.; Escriu, R.; Trujillo, A.J. Ultra-High Pressure Homogenization enhances physicochemical properties of soy protein isolate-stabilized emulsions. Food Res. Int. 75, 357-366 (2015).

30) Ozturk, B.; Argin, S.; Ozilgen, M. Mcclements, D.J. Formation and stabilization of nanoemulsion-based vi- 
tamin E delivery systems using natural biopolymers: Whey protein isolate and gum arabic. Food Chem. 188, 256-263 (2015).

31) Liu, F.; Wang, D.; Ma, C.; Gao, Y. Conjugation of polyphenols prevents lactoferrin from thermal aggregation at neutral pH. Food Hydrocoll. 58, 49-59 (2016).

32) Lam, R.S.; Nickerson, M.T. The effect of $\mathrm{pH}$ and temperature pre-treatments on the structure, surface characteristics and emulsifying properties of alphalactalbumin. Food Chem. 173, 163-170 (2015).

33) Li, B.; Du, W.; Jin, J.; Du, Q. Preservation of (-)-epigallocatechin-3-gallate antioxidant properties loaded in heat treated $\beta$-lactoglobulin nanoparticles. J. Agric. Food Chem. 60, 3477-3484(2012).

34) Caporaso, N.; Genovese, A.; Burke, R.; Barry, R.C.; Sacchi, R. Effect of olive mill wastewater phenolic extract, whey protein isolate and xanthan gum on the behaviour of olive O/W emulsions using response surface methodology. Food Hydrocoll. 61, 66-76 (2016).

35) Li, Y.; Liu, H.; Han, Q.; Kong, K.; Liu, Q. Cooperative antioxidative effects of zein hydrolysates with sage (Salvia officinalis) extract in a liposome system. Food Chem. 222, 74-83(2017).

36) Lee, H.; Yildiz, G.; Santos, L.C.D.; Jianga, S.; Andradea, J.E.; Engeseth, N.J.; Fenga, H. Soy protein nano-aggregates with improved functional properties prepared by sequential pH treatment and ultrasonication. Food Hydrocoll. 55, 200-209 (2016).

37) Shpigelman, A.; Israeli, G.; Livney, Y.D. Thermally-induced protein-polyphenol co-assemblies: beta lactoglobulin-based nanocomplexes as protective nanovehicles for EGCG. Food Hydrocoll. 24, 735-743 (2010).

38) Hasni, I.; Bourassa, P.; Hamdani, S.; Samson, G.; Carpentier, R.; Ali, H.; Riahi, T. Interaction of milk $\alpha$ - and $\beta$-caseins with tea polyphenols. Food Chem. 126, 630639 (2011).

39) Floury, J.; Desrumaux, A.; Legrand, J. Effect of ultrahigh-pressure homogenization on structure and on rheological properties of soy protein-stabilized emulsions. J. Food Sci. 67, 3388-3395(2006).

40) Brown, P.J.; Wright W.B. An investigation of the interactions between milk proteins and tea polyphenols. $J$.
Chromatogr. 11, 504-514(1963).

41) Yangui, A.; Abderrabba, M. Towards a high yield recovery of polyphenols from olive mill wastewater on activated carbon coated with milk proteins: Experimental design and antioxidant activity. Food Chem. 262, $102-$ 109 (2018).

42) Jin, B.; Zhou, X.; Liu, Y.; Li, X.; Mai, Y.; Liao, Y.; Liao, J. Physicochemical stability and antioxidant activity of soy protein/pectin/tea polyphenol ternary nanoparticles obtained by photocatalysis. Int. J. Biol. Macromol. 116, 1-7 (2018).

43) Zhao, L. Physicochemical stability and in vitro bioaccessibility of $ß$-carotene nanoemulsions stabilized with whey protein-dextran conjugates. Food Hydrocoll. 63, 256-264 (2017).

44) Thongkaew, C.; Gibis, M.; Hinrichs, J.; Weiss, J. Polyphenol interactions with whey protein isolate and whey protein isolate-pectin coacervates. Food Hydrocoll. 41, 103-11 (2014).

45) Staszewski, M.V.; Jagus, R.J.; Pilosof, A.M.R. Influence of green tea polyphenols on the colloidal stability and gelation of WPC. Food Hydrocoll. 25, 1077-1084 (2011).

46) Pelton, J.T.; Mclean, L.R. Spectroscopic methods for analysis of protein secondary structure. Anal. Biochem. 277, 167-176 (2000).

47) Kanakis, C.D.; Hasni I.; Bourassa, P.; Tarantilis, P.A.; Polissiou, M.G.; Tajmir, R.H.A. Milk $\beta$-lactoglobulin complexes with tea polyphenols. Food Chem. 127, 1046-1055(2011).

48) Tajmir, R.H.A. An overview of drug binding to human serum albumin: Protein folding and unfolding. Sci. Iran 14, 87-95 (2007)

49) Li, X, Ji N.; Qiu, C.; Xia, M, T.; Xiong, L.; Sun, Q.J. The effect of peanut protein nanoparticles on characteristics of protein- and starch-based nanocomposite films: A comparative study. Ind. Crops Prod. 77, 565-574 (2015).

50) Yerramilli, M.; Longmore, N.; Ghosh, S. Improved stabilization of nanoemulsions by partial replacement of sodium caseinate with pea protein isolate. Food Hydrocoll. 64, 99-111(2017). 